Relations industrielles

Industrial Relations

\title{
The Social Organization of Strikes, by Eric Batstone, Ian Boraston and Stephen Frenkel, Oxford, Warwick Studies in Industrial Relations, 1978, 236 pp.
}

\section{Esther Déom-Camiré}

\section{Volume 34, numéro 2, 1979}

URI : https://id.erudit.org/iderudit/028977ar

DOI : https://doi.org/10.7202/028977ar

Aller au sommaire du numéro

Éditeur(s)

Département des relations industrielles de l'Université Laval

ISSN

0034-379X (imprimé)

1703-8138 (numérique)

Découvrir la revue

Citer ce compte rendu

Déom-Camiré, E. (1979). Compte rendu de [The Social Organization of Strikes, by Eric Batstone, Ian Boraston and Stephen Frenkel, Oxford, Warwick Studies in Industrial Relations, 1978, 236 pp.] Relations industrielles / Industrial

Relations, 34(2), 388-389. https://doi.org/10.7202/028977ar

Tous droits réservés @ C Département des relations industrielles de l'Universite Laval, 1979
Ce document est protégé par la loi sur le droit d'auteur. L'utilisation des services d'Érudit (y compris la reproduction) est assujettie à sa politique d'utilisation que vous pouvez consulter en ligne.

https://apropos.erudit.org/fr/usagers/politique-dutilisation/ 
out on a limb. Here and there, too, the author embraces unwarranted assumptions; for example, he correctly notes that a more positive attitude towards an open door immigration policy in business circles emerged around 1905, but that changed attitude should not be suggested to have produced the ever climbing immigration figures from 1907 to 1914 .

Despite its flaws, this is a most useful study of a neglected subject, and it convincingly demonstrates the dual nature of collective bargaining in the period whereby organized labour became institutionalized into the political and economic structure while at the same time labour in general had limitations placed on the ultimate role it would play in American society.

Sally M. MILLER

University of Warwick

The Social Organization of Strikes, by Eric Batstone, Ian Boraston and Stephen Frenkel, Oxford, Warwick Studies in Industrial Relations, 1978, 236 pp.

Ce livre est le deuxième des auteurs réalisé à partir d'une recherche empirique qu'ils ont effectuée dans une importante usine d'ingénierie en Angleterre. Le premier volume, Shop Stewards in Action, contient des détails sur l'usine et sur les approches conceptuelles et méthodologiques des auteurs qu'il peut s'avérer utile, sinon nécessaire, de lire pour toute personne qui veut tirer le maximum du présent ouvrage.

Les auteurs s'intéressent au processus de mobilisation des travailleurs en vue d'une grève. Leur étude, conduite sur une période de quatre mois, leur a permis d'observer 25 jrs grèves ou situations dans lesquelles des grèves furent évitées de justesse. Ils veulent ici montrer comment les grèves se produisent en mettant l'accent sur les modèles de pouvoir existant parmi les travailleurs. Pour les auteurs, la préparation d'une grève est un processus social qui implique des systèmes d'influence et de pouvoir. Tout en reconnaissant que les grèves, comme forme du conflit industriel, reflètent la dépendance des travailleurs envers l'entreprise et la société en général, ils privilégient cependant l'étude des processus de négociation qui surgissent entre les travailleurs eux-mêmes. Ils s'attachent donc moins aux conditions générales qui favorisent la grève qu'aux conditions spécifiques dans lesquelles elle se réalise. Ils choisissent par le fait même de laisser de côté le rôle de la direction sur le déclenchement d'une grève.

L'ouvrage est divisé en deux parties. La première est consacrée à l'étude des relations de pouvoir existant parmi les travailleurs et par lesquelles les mouvements de grève furent encouragés ou contenus. Les auteurs s'attardent d'abord à différencier la grève de d'autres types d'action collective. Cela les amène ensuite à expliquer pourquoi la direction ne définit pas toujours les arrêts de travail comme des grèves: en général, les arrêts de travail où les travailleurs ne quittent pas l'usine et où la situation, au moment même ou après l'action, est considérée comme légitime par la direction ne sont pas classés comme grèves. Il s'ensuit donc que les arrêts de travail initiés par les travailleurs sont plus fréquents que les statistiques de grève ne le suggèrent.

Les auteurs considèrent ensuite les conditions qui facilitent la grève. Ils se limitent cependant aux facteurs liés à la technologie et à l'organisation des groupes de travail, ce qui les amène à parler du phénomène du pouvoir, ainsi qu'aux conditions institutionnelles et organisationnelles.

Les 3 chapitres suivants sont consacrés à l'étude plus détaillée du processus de mobilisation en vue d'une grève: les auteurs se concentrent sur le vocabulaire particulier qui s'associe à une grève éventuelle; en se dirigeant vers la grève, les travailleurs et leurs officiers syndicaux se créent une «logique», un «rationnel» pour justifier leur action. Les auteurs examinent ainsi le vocabulaire utilisé autant pour s'opposer à une grève que pour la supporter et autant du point de vue individuel que collectif (chap. 4). 
Les auteurs traitent ensuite d'un autre aspect du processus de mobilisation i.e. les «acteurs» qui supportent ou s'opposent aux initiatives en vue d'une grève. Ainsi, ils identifient certains types de travailleurs ou d'officiers syndicaux qui ont des capacités supérieures à la moyenne pour initier des mouvements de grève: le "griever» et le «opinion leader" parmi les travailleurs et le "leader» parmi les différents types de délégués syndicaux. Les auteurs décrivent aussi les réseaux de communication qui existent entre ces catégories de travailleurs et d'officiers syndicaux (chap. 5).

Le chapitre 6 intègre les éléments des deux chapitres précédents en étudiant la dynamique du mouvement de grève à travers l'exercice du pouvoir et l'emploi d'un vocabulaire particulier par les différents acteurs.

La deuxième partie examine en détail la préparation d'une grève générale dans l'usine à cause d'une tentative de l'entreprise pour changer le système de paiement, lors des négociations annuelles. Les auteurs insistent surtout sur le rôle du leadership et des réseaux d'influence des délégués syndicaux sur le développement d'un degré de conscience collective parmi les membres. La majeure partie de cette deuxième section est donc consacrée au processus de mobilisation mis en place en vue d'une grève qui, finalement, n'aura pas lieu.

Les auteurs insistent en conclusion sur l'importance de comprendre les réseaux de pouvoir et d'influence parmi les travailleurs dans l'analyse du processus de mobilisation. Ils se disent aussi d'accord avec les théories qui soutiennent que la propension à la grève reflète une variété de facteurs institutionnels et organisationnels. "At a general level such theories point to the conditions under which strikes are likely to occur. But strikes involve groups of workers making common decisions to stop work, and the dynamics of this process are too frequently given little attention. In this volume we have looked at how workers negotiate together over the strategies they should employ" (p. 223)
En somme, l'ouvrage de Batstone, Boraston et Frenkel constitue une très bonne recherche pour tous ceux qui s'intéressent à la grève, comme forme du conflit industriel. Il s'agit d'une étude pratique abondamment appuyée de statistiques et illustrée d'exemples bien concrets tirés d'une situation que les auteurs ont manifestement appris à connaître en profondeur.

\section{Esther DÉOM-CAMIRẼ}

Université Laval

S'adapter à un monde en pleine évolution. Choix de textes sur la qualité de la vie au travail, par George F. Sanderson (ed.), Ottawa, La Gazette du travail, édition spéciale, 1978, $93 \mathrm{pp}$.

Cet ouvrage regroupe une dizaine de courts textes déjà parus concernant la qualité de la vie au travail. On y retrouve une étude du concept de qualité de vie au travail (QVT) ainsi que la description de nombreuses expériences de QVT tentées avec succès au Canada et à l'étranger. Il s'agit d'un ouvrage de vulgarisation destiné à un large public et écrit dans un langage accessible.

Dès l'avant-propos, les questions de fond sont soulevées: "les gestionnaires sont en train de découvrir que plusieurs des solutions aux problèmes du personnel, autrefois infaillibles, ne sont plus efficaces... Un sentiment d'aliénation croissant se manifeste parmi la nouvelle génération dont l'expérience et les espérances sont différentes de celles de travailleurs ayant vécu à une époque de parcimonie, lorsque les récompenses d'ordre économique primaient sur tout». Le problème est bien posé.

Le premier texte présenté dans le recueil est signé par le Ministre du travail du Canada. Il s'agit d'une prise de position très ferme du gouvernement fédéral en faveur d'une amélioration substantielle de la qualité de la vie au travail. Le vocabulaire utilisé ne laisse place à aucun doute et le ton est catégo- 